\title{
Reaction-field calculation for the quantum Potts model
}

\author{
R Dekeyser $\dagger$, A Maritan $\ddagger$ and A L Stella \\ $\div$ Instituut voor Theoretische Fysika, K U Leuven, B-3030 Heverlee. Belgium \\ $\doteqdot$ Dipartimento di Fisica e INFN, Università di Padova. Italy \\ § Dipartimento di Fisica e Unità GNSM del CNR. Università di Padova. Italy
}

Received 10 January 1984

\begin{abstract}
The reaction-field approximation is applied to the $d$-dimensional $q$-state quantum Potts model in an external transverse field. With the exception of $d \geqslant 3$, the tricritical value $q_{c}(d)$, below which the system has a second-order phase transition. decreases with increasing external field strength. From the ground state of the quantum $d$-dimensional model. an improved value of $q_{\mathrm{c}}$ is obtained for the classical model in $d+1$ dimensions. A direct explanation is also obtained for the expected tricritical point in finite-thickness slabs of cubic ferromagnets (like $\mathrm{DyAl}_{2}$ ) with a field in the (111) direction.
\end{abstract}

\section{Introduction}

In a previous paper (Dekeyser et al 1983) we have solved the general $d$-dimensional $q$ state Potts model on a hypercubic lattice in Onsager's reaction-field approximation. This approximation is an improvement on the molecular-field approximation (MFA), in the sense that it takes into account the direct influence on neighbouring sites of the particular state in which a given site may be found. More specifically, we focused our attention on the prediction for $q_{\mathrm{c}}(d)$, giving the borderline between first- and secondorder transitions. As is well known, a simple MFA gives the completely wrong prediction $q_{c}(d)=2$ for this tricritical line. The result of our reaction-field approach was a remarkable qualitative improvement for $q_{\mathrm{c}}(d)$.

In the present paper we apply the same method to the quantum mechanical case of the $q$-state Potts model in an external transverse field (Kogut and Sinclair 1981). The basic ideas of our derivation are equivalent to those of the calculation for the classical Potts system. The presence of the transverse field, however, complicates the necessary computational effort a great deal. Instead of reproducing these calculations in full detail, we will mainly stress the differences from the previous derivation due to the quantum mechanical nature of the Potts model in a transverse field. From the methodological point of view, this paper is a demonstration of how to apply the reaction-field idea to a quantum model.

After summarising the main formulae for the model and its MFA in $\S 2$, the reaction-field approach is described in $\S 3$. In $\$ 4$ we discuss a few applications of our calculation. First of all, from the ground-state result we may derive an improved prediction for $q_{c}(d+1)$ for the classical zero-field model. Next we discuss the cubic ferromagnets such as $\mathrm{DyAl}_{2}$ with a magnetic field in the (111) direction as a possible physical 
realisation of the quantum three-state model. This allows us to explain the possible existence of tricritical points for slabs of finite thickness of these materials, not in terms of a dimensional crossover, but simply in terms of quantum effects.

\section{Description of the model and MFA}

When a transverse field is switched on, the $q$-state Potts model becomes a quantum model. A variable $s_{i}$ is associated with each site $i$ of a regular (hypercubic) lattice, where $s_{i}$ may assume the integer values from 1 to $q$. Accordingly, we associate with each site a $q$-component column state vector $s_{i}$ such that

$$
\left(s_{i}\right)_{j}=\delta_{j k} \quad \text { when } s_{i}=k(k=1, \ldots, q) .
$$

The Hamiltonian of the model may be written as

$$
\mathscr{H}=-J \sum_{\langle i j\rangle} \boldsymbol{P}_{i} \cdot \boldsymbol{P}_{j}-h \sum_{i} B_{i} .
$$

As usual, the first sum runs over nearest-neighbour pairs $\langle i j\rangle$. The $q$ components $P_{i}^{k}$ of the vector $\boldsymbol{P}_{i}$ are the projectors on the $k$ th Potts state at site $i$ :

$$
\left(P_{i}^{k}\right)_{m n}=\delta_{m k} \delta_{n k} \text {. }
$$

The first term of the Hamiltonian thus assigns an energy $-J$ to every set of neighbouring sites that are in the same Potts state. The transverse-field strength $h$ is combined with the operators $B_{i}$ where

$$
\left(B_{i}\right)_{m n}=1-\delta_{m n}
$$

The transverse field thus tries to prevent condensation in any particular state.

Below a certain temperature and/or below a certain field strength we expect to find a condensation into, for example, the state $s=1$, such that the thermal averages of the projectors are given by

$$
\begin{aligned}
& p_{1}=\left\langle P_{i}^{1}\right\rangle=[1+(q-1) m] / q \\
& p_{n}=\left\langle P_{i}^{n}\right\rangle=(1-m) / q \quad 2 \leqslant n \leqslant q .
\end{aligned}
$$

$m$ is the order parameter. The MFA for this model is derived from the effective one-site Hamiltonian $\mathscr{H}_{\text {MFA }}$, which one obtains by replacing in (1) the projectors $P_{j}^{k}$ on the $z$ ( $=2 d$ for hypercubic lattices) neighbours $j$ of a particular site $i$ by their expectation values (4):

$$
\mathscr{H}_{\mathrm{MFA}}=-J z\left(p_{1} P^{1}+\sum_{n=2}^{q} p_{n} P^{n}\right)-h B .
$$

Defining (with $\beta=1 / k T$ )

$$
-\beta \mathscr{H}_{\mathrm{MFA}}=H
$$

we thus obtain the matrix elements

$$
\begin{array}{ll}
H_{11}=\beta J z p_{1} \equiv \alpha & \\
H_{n n}=\beta J z p_{n} \equiv \gamma & 2 \leqslant n \leqslant q \\
H_{k l}=\beta h \equiv \eta & 1 \leqslant j \neq k \leqslant q .
\end{array}
$$


The relevant properties of the eigenvalues and eigenvectors of this matrix $H$ are as follows.

(i) There are two eigenvectors $\boldsymbol{e}_{+}$and $\boldsymbol{e}_{-}$with a non-zero first component. They belong to the eigenvalues $\lambda_{+}$and $\lambda_{-}$respectively, with

$$
\lambda_{ \pm}=\alpha+u \pm w
$$

where

$$
\begin{aligned}
& u=\frac{1}{2}[\gamma-\alpha+(q-2) \eta] \\
& w=\left[u^{2}+(q-1) \eta^{2}\right]^{1 / 2} .
\end{aligned}
$$

The (not yet normalised) eigenvectors have components

$$
\begin{aligned}
& \left(e_{ \pm}\right)_{1}=(q-1) \eta \\
& \left(e_{ \pm}\right)_{n}=u \pm w \quad 2 \leqslant n \leqslant q .
\end{aligned}
$$

(ii) The third eigenvalue

$$
\lambda_{0}=\gamma-\eta
$$

is $(q-2)$-fold degenerate. Its eigenspace is formed by all the vectors $f$ with the properties

$$
f_{1}=0 \quad \text { and } \quad \sum_{n=2}^{q} f_{n}=0
$$

(iii) The unitary operator transforming from the standard Potts-state basis to the basis of eigenstates of $H$ therefore has the following properties:

$$
\begin{aligned}
& T_{11}=(q-1) \eta C_{+} \\
& T_{12}=(q-1) \eta C_{-} \\
& T_{1 n}=0 \quad 3 \leqslant n \leqslant q \\
& \left.\begin{array}{l}
T_{n 1}=(u+w) C_{+} \\
T_{n 2}=(u-w) C_{-}
\end{array}\right\} \quad 2 \leqslant n \leqslant q
\end{aligned}
$$

where

$$
C_{ \pm}=\left[(q-1)^{2} \eta^{2}+(q-1)(u \pm w)^{2}\right]^{-1 / 2} .
$$

The matrix elements not defined by (14) depend on the particular choice made for the $(q-2)$-fold-degenerate eigenstates. We will not need a precise knowledge about these states, since we will use the unitarity of $T$, expressed by ( $T$ has real elements)

$$
\sum_{n=3}^{q} T_{k n} T_{l n}=\delta_{k l}-T_{k 1} T_{l 1}-T_{k 2} T_{n} .
$$

Let us come back now to the MFA. From (5) we obtain the self-consistency condition

$$
\begin{aligned}
p_{1} & =Z^{-1} \operatorname{Tr}\left(P^{1} \mathrm{e}^{H}\right) \\
& =Z^{-1}\left(T_{11}^{2} \mathrm{e}^{\lambda_{-}}+T_{12}^{2} \mathrm{e}^{\lambda_{-}}\right)
\end{aligned}
$$


where the single-site partition function is

$$
Z=\mathrm{e}^{\lambda-}+\mathrm{e}^{\lambda_{-}}+(q-2) \mathrm{e}^{\lambda_{0}} .
$$

Equation (17) is the standard MFA equation of state for this model, giving in principle the order parameter $m$ as a function of $\beta J z, \eta=\beta h$ and $q$. The right-hand side of (17) can indeed be written as a function of the difference

$$
\alpha-\gamma=\beta J z m \text {. }
$$

By a series expansion, (17) then reduces to an equation

$$
g(m) \equiv a_{1} m+a_{2} m^{2}+\ldots=0 .
$$

This may be interpreted as the derivative of the free energy with respect to $m$. As is well known from the Landau theory, a second-order phase transition occurs when $a_{1}=0$ and $a_{2}>0$ (if only positive $m$ are allowed, see Dekeyser et al 1983), while $a_{2}<0$ means that the system underwent a first-order transition. The tricritical point is then determined from $a_{1}=0=a_{2}$. One easily verifies that for all values of $h$ the approximation (17) suffers from the same fault as in the zero-field case: the transition is first order for all $q>2$.

\section{The reaction-field approximation}

In order to obtain the reaction-field approximation for this model, one has to replace the expectation values $p_{k}$ in the effective single-site Hamiltonian (5) by the conditional expectation values $p_{k}^{\mathrm{c}}$. These quantities give the thermal expectation value for finding site $j$ in state $k$, under the condition that one of its $z$ neighbours, $s_{i}$, is fixed in state $k$ :

$$
p_{k}^{c}=\left.\left\langle P_{j}^{k}\right\rangle\right|_{s_{i}=k} .
$$

An exact calculation of these conditional expectation values would be equivalent to solving the model exactly. We will, however, limit ourselves to an approximate calculation, in which, starting from the MFA, the effect of fixing $s_{i}$ in state $k$ is taken into account in linear order in the quantities $\left(p_{k}^{c}-p_{k}\right)$. From a physical point of view this amounts to taking into account only the conditioning effects which propagate directly between nearest-neighbour sites; a higher-order calculation would also require us to take into account effects propagating through intermediate sites in the lattice.

The reaction-field calculation then runs parallel to the calculation of $\S 2$, except for the fact that in (7) and subsequent equations we have to use

$$
\begin{aligned}
& \alpha^{\prime}=\beta J z p_{1}^{\mathrm{c}} \\
& \gamma^{\prime}=\beta J z p_{n}^{\mathrm{c}} \quad M 2 \leqslant n \leqslant q
\end{aligned}
$$

instead of $\alpha$ and $\gamma$. The MFA Hamiltonian (5) is thus replaced by the reaction-field Hamiltonian

$$
\mathscr{H}_{\mathrm{R}}=-J z\left(p_{1}^{\mathrm{c}} P^{1}+\sum_{n=2}^{q} p_{n}^{\mathrm{c}} P^{n}\right)-h B .
$$

The main problem resides in the calculation of the quantities $\alpha^{\prime}$ and $\gamma^{\prime}$, or rather of their difference $\alpha^{\prime}-\gamma^{\prime}=\beta J z\left(p_{1}^{\mathrm{c}}-p_{n}^{\mathfrak{c}}\right)$.

The calculation of $p_{1}^{c}$ is rather simple, because this quantity can be obtained as the average of $P^{1}$ corresponding to the effective Hamiltonian (23), modified in such a way 
that one of the neighbours is definitely fixed in state 1 . Thus $p_{1}^{c}$ can be obtained from equation (17), provided that $\alpha$ and $\gamma$ are substituted for by

$$
\begin{gathered}
\alpha_{1}=\beta J(z-1) p_{1}^{\mathrm{c}}+\beta J=\alpha^{\prime}+\beta J\left(1-p_{1}^{\mathrm{c}}\right) \\
\gamma_{1}=\beta J(z-1) p_{n}^{\mathrm{c}}=\gamma^{\prime}-\beta J p_{n}^{\mathrm{c}} .
\end{gathered}
$$

This would provide an explicit expression for $p_{1}^{\mathfrak{c}}$ as a function of itself and the as yet unknown value of $p_{n}^{c}$. Since we will, in any case, resort to a linearisation in the $p_{k}^{c}-p_{k}$ in order to solve this equation and the analogous one for $p_{n}^{c}$, we will immediately use a first-order perturbation method for calculating the conditional probabilities. The conditional Hamiltonian for $p_{1}^{c}$ is given by

$$
\mathscr{H}_{1}^{c}=\mathscr{H}_{\mathrm{R}}+V_{1}
$$

where

$$
\begin{aligned}
V_{1} & =J\left(p_{1}^{\mathrm{c}}-1\right) P^{1}+J \sum_{n=2}^{q} p_{n}^{\mathrm{c}} P^{n} \\
& =J p_{n}^{\mathfrak{c}}+J\left(p_{1}^{\mathfrak{c}}-1-p_{n}^{\mathfrak{c}}\right) P^{1} .
\end{aligned}
$$

We used the fact that for $n$ and $n^{\prime}$ larger than one, we have

$$
p_{n}^{c}=p_{n}^{c}
$$

and furthermore

$$
\sum_{k=1}^{q} P^{k}=1
$$

In the expression

$$
p_{1}^{c}=\operatorname{Tr}\left[P^{1} \exp \left(-\beta \mathscr{H}_{1}^{c}\right)\right]\left[\operatorname{Tr} \exp \left(-\beta \mathscr{H}_{1}^{c}\right)\right]^{-1}
$$

we will now twice use the expansion

$\exp \left(-\beta \mathscr{H}_{1}^{c}\right)=\exp \left(-\beta \mathscr{H}_{\mathrm{R}}\right)\left(1-\int_{0}^{\beta} \mathrm{d} \mu \exp \left(\mu \mathscr{H}_{\mathrm{R}}\right) V_{1} \exp \left(-\mu \cap \mathrm{h}_{\mathrm{R}}\right)+\ldots\right)$

in order to obtain, up to first order in $V_{1}$ :

$p_{1}^{c}=p_{1}\left(1+\beta\left\langle V_{1}\right\rangle\right)-\left\langle\int_{0}^{\beta} \mathrm{d} \mu \exp \left(\mu \mathscr{H}_{\mathrm{R}}\right) V_{1} \exp \left(-\mu \mathscr{H}_{\mathrm{R}}\right) P^{1}\right\rangle$.

The expectation values $\langle\ldots\rangle$ in this expression are thermal expectation values with respect to the Hamiltonian $\mathscr{H}_{\mathrm{R}}$. The first term in $V_{1}$, being just a $c$-number, does not contribute to $p_{1}^{\mathrm{c}}$.

A similar procedure may be followed for $p_{n}^{c}$. The conditional Hamiltonian, for example, for $p_{2}^{\mathrm{c}}$ is

$$
\mathscr{H}_{2}^{\complement}=\mathscr{H}_{\mathrm{R}}+V_{2}
$$

where

$$
\begin{aligned}
V_{2} & =J p_{1}^{c} P^{1}+J\left(p_{2}^{c}-1\right) P^{2}+J \sum_{n=3}^{q} p_{n}^{c} P^{n} \\
& =J p_{n}^{c}+J\left(p_{1}^{c}-p_{n}^{c}\right) P^{1}-J P^{2}
\end{aligned}
$$


and a formula analogous to (31) yields $p$.

Combining the expression thus obtained, we finally obtain:

$$
\begin{gathered}
p_{1}^{c}-p_{n}^{c}=p_{1}-p_{n}+\beta J\left(p_{1}-p_{n}\right)\left[p_{1}\left(p_{1}-p_{n}\right)-\left(p_{1}+p_{n}\right)\right. \\
\left.-\left(X_{11}-X_{1 n}\right)\right]+\beta J\left(X_{11}-X_{n n}\right)
\end{gathered}
$$

where again $n>1$, and

$$
X_{i j} \equiv \frac{1}{\beta} \int_{0}^{\beta} \mathrm{d} \mu\left\langle\exp \left(\mu \mathscr{H}_{\mathrm{R}}\right) P^{i} \exp \left(-\mu \mathscr{H}_{\mathrm{R}}\right) P^{i}\right\rangle .
$$

We now have two relations between the order parameter $m$ and $\alpha^{\prime}-\gamma^{\prime}=$ $\beta J z\left(p_{1}^{\mathfrak{c}}-p_{n}^{\mathfrak{c}}\right)$. Equation (17), adapted for the reaction-field Hamiltonian (23), yields a series expansion of $m$ in powers of $\alpha^{\prime}-\gamma^{\prime}$. Analogous series expansions may be obtained for $X_{11}-X_{1 n}$ and $X_{11}-X_{n n}$. Equation (34) thus establishes a second independent relation between $m$ and $\alpha^{\prime}-\gamma^{\prime}$. Eliminating $\alpha^{\prime}-\gamma^{\prime}$ between (17) and (34) gives us a reaction-field equation of the form (20). The simultaneous vanishing of the coefficients $a_{1}$ and $a_{2}$ of the linear and quadratic terms of this series expansion determines the tricritical point.

In this way, we have determined numerically the line of tricritical points in the $(q, d)$-plane for several values of $\eta$. The results are illustrated in figure 1 .

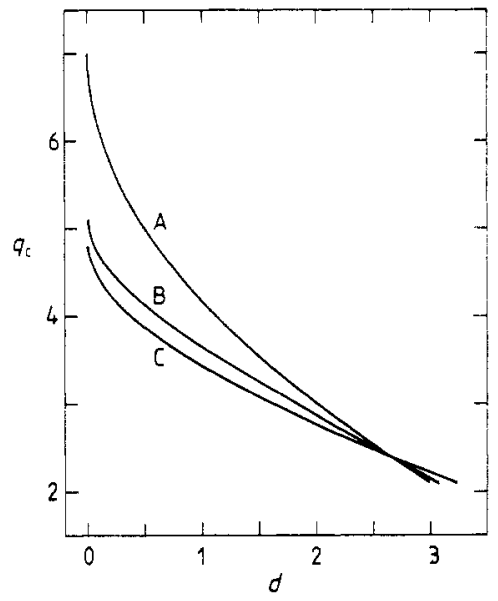

Figure 1. The line of tricritical points in the $(q, d)$-plane for: $\mathrm{A}, \eta=0 ; \mathrm{B}, \eta=1 ; \mathrm{C}, \eta=\infty$ $(\eta=h / k T)$.

\section{Discussion}

The results for $h=0$ are obviously identical with the classical results obtained (with a single-site reaction-field approximation) in our previous paper (Dekeyser et al 1983). The other extreme situation, $\eta=\infty$, may be viewed as a ground-state calculation $(\beta=$ $\infty)$ for the Potts model in a transverse field. The ground-state phase transition in the transverse Ising model is a special example $(q=2)$, and is well known to be of second order. For general $q$, the simple MFA would predict that the transition in the ground 
state of the transverse Potts model is first order as soon as $q>2$, but our reaction-field result gives a much more realistic prediction for the borderline between first- and second-order transitions.

On the other hand, it is known (Kogut 1979) that the ground state of a $d$-dimensional quantum system with transverse field is equivalent to the finite-temperature $D$-dimensional classical system without a field, with $D=d+1$. Since this equivalence is based on a transfer matrix treatment of the extra dimension, our $\eta=\infty$ results may also be interpreted as an improvement on the reaction-field calculation for the classical Potts model without a field. The reaction-field is then effectively working between infinite one-dimensional chains of lattice sites, and this must be expected to be a better approximation than the single-site reaction-field. From this point of view, we understand why the general trend (for $d \leqslant 3$ ) of our result is a shift of $q_{\mathrm{c}}(d)$ towards lower values when $\eta$ increases from zero to infinity: it is the result of shifting the $q_{c}(d)$ curve to the left. Ideally we would expect $q_{\mathrm{c}}(d, \eta=0)=q_{\mathrm{c}}(d-1, \eta=\infty)$. The observed shift in dimensionality is, however, smaller than unity. This may be understood from the fact that our classical single-site reaction-field calculation underestimated the tricritical dimensionality corresponding to a certain value of $q$ (see Dekeyser et al 1983). Any improvement on this approximation is thus expected to shift the curve towards higher values of $d$. The approximation obtained here for $\eta=\infty$ is then the superposition of (i) the above mentioned shift of the $q_{\mathrm{c}}(d)$ curve from $d$ to $d-1$, and (ii) a shift to the right due to the implicit exact transfer matrix treatment of one dimension. We also believe that the crossing of the curves for $d \geqslant 3$ is caused by the growing importance of effect (ii). In this way we now obtain from our $\eta=\infty$ calculation the following approximations for the classical Potts model: $q_{\mathrm{c}}(4) \simeq 2.219, q_{\mathrm{c}}(3) \approx 2.764$ and $q_{\mathrm{c}}(2)=3.428$ (exact value: 4.0 ). Our value for $q_{c}(3)$ is in good agreement with the estimate obtained by Kogut and Sinclair (1982) on the basis of a 1/q-expansion.

Even if the quantitative results are not very accurate, there is an interesting qualitative prediction in our calculation. For a certain range of $q$ - and $d$-values we have shown that, by increasing the strength of a transverse field, it is possible to change the nature of the phase transition from second order to first order. As a numerical example, a two-dimensional Potts system with $q=2.9$ in zero field is predicted to have a secondorder phase transition in our approach, whereas it has a first-order transition in strong fields. The tricritical point would be found at $h / J \simeq 0.816$ and $\beta J \simeq 0.98$. Taking into account the estimated deviation of our results from the true values in zero field, it may be expected that in reality such a phenomenon will also occur for $d=2, q=3$.

In this sense our calculation gives a direct description of the tricritical point that is expected to be found in a finite slab of a cubic ferromagnet such as $\mathrm{DyAl}_{2}$ by increasing the magnetic field along the (111) direction (Shnidman and Domany 1981). Such a system is supposed to be a realisation of the $d=2, q=3$ model with the three Potts states corresponding to magnetised states along the three easy axes (100), (010) and (001), with a second-order phase transition (Mukamel et al 1976, Barbara et al 1978). The effect of the (111) field, apart from creating the easy axes that correspond to the three Potts states, is to add to the Hamiltonian a transverse-field term as described by our formula (3). Indeed, the Larmor precession induced on a magnetic moment by an external field corresponds in our discretised three-state Potts model to the transition probabilities caused by the second term of Hamiltonian (1). Our calculation then suggests that the phase transition for such a $d=2$ model is indeed of second order up to a particular field strength, above which a first-order transition should be seen (see figure 2). 


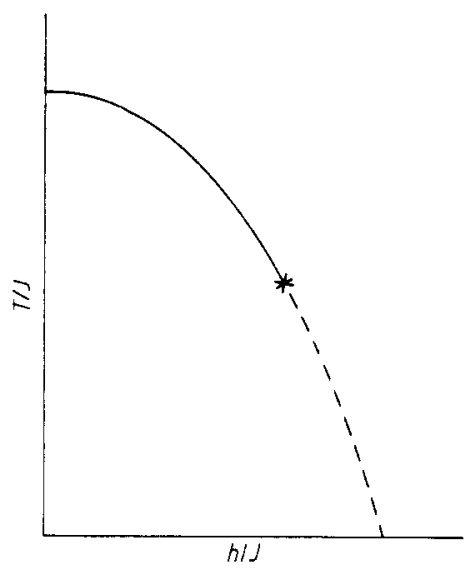

Figure 2. Qualitative phase diagram expected for the two-dimensional three-state quantum Potts model. The full and broken curves represent second-and first-order transitions respectively.

The previously mentioned authors are of course correct when claiming that an increasing transverse field corresponds to an increase of the effective dimensionality. We have already mentioned the fact that the system behaves like a $(d+1)$-dimensional system when $\eta$ becomes infinite. Finite values of $\eta$ then correspond to a finite number of $d$-dimensional layers, forming a slab of finite thickness in the $(d+1)$-dimensional space (Hertz 1976). In this sense, our calculation provides a very simple approach to the expected tricritical point for a thin slab of the three-state Potts model (Andelman and Berker 1981, Shnidman and Domany 1981). Since increasing the transverse-field strength corresponds effectively to a growing slab thickness, this is a special case of dimensional crossover, due to quantum effects.

\section{References}

Andelman A and Berker A N 1981 J. Phys. A: Math. Gen. 14 L91

Barbara B, Rossignol M F and Bak P 1978 J. Phys. C: Solid State Phys. 11 L183

Dekeyser R, Maritan A and Stella A L 1983 J. Phys. A: Math. Gen. 161713

Hertz J A 1976 Phys. Rev. B 141165

Kogut J B 1979 Rev. Mod. Phys. 51659

Kogut J B and Sinclair D K 1981 Phys. Lett. 81A 149

1982 Solid State Commun. 41187

Mukamel D, Fisher M E and Domany E 1976 Phys. Rev. Lett. 37565

Shnidman Y and Domany E $1981 \mathrm{~J}$. Phys. C: Solid State Phys. $14 \mathrm{~L} 773$ 